Meta

Journal des traducteurs

Translators' Journal

\title{
Intertextualité dans la traduction des albums de type « double lectorat "
}

\section{Sungyup Lee}

Volume 60, numéro 1, avril 2015

URI : https://id.erudit.org/iderudit/1032411ar

DOI : https://doi.org/10.7202/1032411ar

Aller au sommaire du numéro

\section{Éditeur(s)}

Les Presses de l’Université de Montréal

ISSN

0026-0452 (imprimé)

1492-1421 (numérique)

Découvrir la revue

Citer cet article

Lee, S. (2015). Intertextualité dans la traduction des albums de type « double lectorat ». Meta, 60(1), 53-70. https://doi.org/10.7202/1032411ar

\section{Résumé de l'article}

Cet article traite de la question de l'intertextualité dans la traduction des albums destinés à un double lectorat. Le lecteur enfant et le lecteur adulte, qui constituent le double lectorat, ont deux compétences encyclopédiques différentes relativement à l'actualisation textuelle. L'intertextualité est une stratégie textuelle qui laisse apercevoir une corrélation entre la double réception et l'étendue du bagage du lecteur. Le rapport intertextuel entraîne une relation d'inclusion ou d'exclusion quant au double lectorat, selon qu'il fait partie de la culture de l'adulte ou de celle de l'enfant. Ainsi, le transfert du réseau intertextuel consiste à appréhender l'écart encyclopédique entre les deux lecteurs de la culture d'origine et à faire passer cette distance dans la culture de réception, en vue de reconstruire un texte assurant à son tour le double niveau de réception. 


\title{
Intertextualité dans la traduction des albums de type «double lectorat»
}

\author{
SUNGYUP LEE \\ Université d'Ewha, Séoul, Corée du Sud \\ sungyuplee@hotmail.com
}

\begin{abstract}
RÉSUMÉ
Cet article traite de la question de l'intertextualité dans la traduction des albums destinés à un double lectorat. Le lecteur enfant et le lecteur adulte, qui constituent le double lectorat, ont deux compétences encyclopédiques différentes relativement à l'actualisation textuelle. L'intertextualité est une stratégie textuelle qui laisse apercevoir une corrélation entre la double réception et l'étendue du bagage du lecteur. Le rapport intertextuel entraine une relation d'inclusion ou d'exclusion quant au double lectorat, selon qu'il fait partie de la culture de l'adulte ou de celle de l'enfant. Ainsi, le transfert du réseau intertextuel consiste à appréhender l'écart encyclopédique entre les deux lecteurs de la culture d'origine et à faire passer cette distance dans la culture de réception, en vue de reconstruire un texte assurant à son tour le double niveau de réception.
\end{abstract}

\begin{abstract}
This article deals with the question of intertextuality in the translations of crossover picture books. Child and adult readers, who constitute dual readership, have different levels of encyclopedic competence in terms of textual actualization. Intertextuality, as one textual mechanism, shows that there is a correlation between dual reception and the background knowledge of readers. An intertextual connection results in either an inclusive or exclusive relationship in terms of dual readership. Therefore, the translation of allusions in such picture books means to scrutinize the encyclopedic distance between two readerships: the culture of origin and the culture of reception. In order to produce a target text, which may remain a crossover text, this gap must be conveyed accordingly.
\end{abstract}

\section{MOTS-CLÉS/KEYWORDS}

double lectorat, intertextualité, culture, album, traduction

dual address, intertextuality, culture, picturebook, translation

\section{Introduction}

Depuis «la mort de l'auteur» (Barthes 1968), le texte occupe le devant de la scène. Contrairement à l'approche littéraire du XIX siècle, dont Lanson (1894) est une figure représentative, la critique littéraire ne considère plus l'auteur comme l'unique porteur de sens; elle postule une certaine autonomie du texte. Indépendamment de l'intention de son producteur, le texte est mis en relation avec d'autres pour permettre la construction du sens. Par la suite, les spécialistes de la réception et de la lecture, comme Jauss (1978), Iser (1985) et Eco (1985), mettent en valeur l'appropriation du texte par le lecteur: ils arrachent ainsi la lecture à la passivité. Selon Iser (1985: 48), «le lieu de l'œuvre littéraire est donc celui où se rencontrent le texte et le lecteur». Le texte et le lecteur ne sont pas indépendants l'un de l'autre, mais dans une relation d'interdépendance. Plus précisément, l'écriture est tendue vers le lecteur et la lecture est une perception guidée. 
La place accordée au lecteur est davantage visible en littérature jeunesse, en ce sens que la désignation du genre laisse voir que cette écriture littéraire est destinée à un type de lecteur bien précis. Nières-Chevrel (1984: 80) affirme ainsi qu'«au sens strict, la littérature pour la jeunesse est tout entière une littérature adaptée, c'est-à-dire définie par son public. Les adultes produisent une littérature adaptée à un public perçu comme différent, qui requiert donc des conduites spécifiques». Depuis une vingtaine d'années, la question du lecteur dans ce domaine littéraire est pourtant envisagée dans une nouvelle direction: la littérature jeunesse attise la convoitise du lecteur enfant, mais également du lecteur adulte. Le texte est équivoque et l'écriture est tendue vers ce double lectorat. Comment le texte parvient-il à séduire le lectorat naïf sans ennuyer le lectorat chevronné, et vice versa? Quelles stratégies d'écriture rendent cela possible?

Le lecteur reste toujours une question aussi importante en traduction que dans l'écriture littéraire. L'expérience de lecture par le sujet traduisant a toujours lieu avant une réécriture adaptée au lecteur de la traduction. Plassard (2007: 21) souligne que «Traduire, c'est dès lors lire un écrit préexistant dans la finalité particulière de le réécrire». La lecture est déjà inscrite dans la réécriture que le traducteur propose à son propre lecteur. En effet, le destinataire du texte d'arrivée est ancré dans la culture de réception, c'est-à-dire dans une encyclopédie différente de celle de l'original. L'encyclopédie (Eco 1985: 95-106) est un ensemble de connaissances, d'idéologies, d'expériences (y compris d'expériences de lecture) qui interviennent dans le processus de signification, en l'occurrence dans toutes approches d'un texte. Doté d'une compétence encyclopédique appropriée, le traducteur se met d'abord dans la peau du lecteur du texte original afin de saisir l'effet de lecture le plus amplement possible; puis il se met au travail de reformulation en anticipant le profil du lecteur du texte d'arrivée. Le traducteur, qui semble avant tout avoir pour tâche de réécrire, se place d'abord en tant que lecteur de la culture d'origine pour disséquer l'original et ensuite, se met à la place du lecteur de la culture de réception afin de produire une traduction compréhensible. Le lecteur est alors aussi essentiel que le texte et son créateur.

Cet article a pour but d'aborder la question du double lectorat dans la traduction des albums. On se penchera sur l'intertextualité, stratégie textuelle qui incite à adopter un double niveau de lecture, ainsi que sur son traitement en traduction.

\section{Double lectorat et intertextualité}

Le double lectorat, composé de lecteurs enfants et de lecteurs adultes, est un phénomène qui existe depuis longtemps. On peut citer quelques titres d'ouvrages rappelant ce double lectorat: Les Contes de la mère l'Oye ou Histoires et contes du temps passé (1697) de Charles Perrault ${ }^{1}$, Voyages de Gulliver (1726) de Jonathan Swift ${ }^{2}$, Alice au pays des merveilles (1865) de Lewis Carroll'3, Vendredi ou la Vie sauvage (1971) de Michel Tournier ${ }^{4}$. Ce phénomène littéraire n'est pas nouveau, néanmoins sa théorisation est relativement récente: depuis les années 1990, le double lectorat retient l'attention de spécialistes, notamment Wall (1991: 35-36), Kümmerling-Meibauer (1999: 15-17) et Beckett (2011 : 59). Il concerne les textes littéraires qui voyagent entre lecteur enfant et lecteur adulte: il peut s'agir soit d'un seul et même texte destiné aux deux types de lecteurs, soit d'un texte visant initialement l'un d'entre eux, réécrit à 
destination de l'autre. À titre d'illustration, si Perrault a produit des contes qui s'adressent simultanément aux deux types de lecteurs, Tournier a d'abord écrit Vendredi ou les Limbes du Pacifique (1967) pour le lectorat adulte, puis l'a adapté pour le lectorat enfant sous le titre de Vendredi ou la Vie sauvage (1971). Si un texte est écrit pour un lectorat et est réécrit pour un autre, chacune des deux productions textuelles vise un lectorat homogène en termes d'âge. Par contre, le texte sous une seule et même forme, destiné à deux lectorats à la fois, matérialise l'ouverture textuelle d'autant mieux qu'il présuppose originairement deux niveaux de réception. En effet, chacun de ces deux lectorats dispose, du fait de l'âge, de compétences cognitives et encyclopédiques différentes, entraînant des appropriations du texte également différentes. Cela étant posé, cet article va porter sur les textes destinés à la fois au jeune lecteur et au lecteur chevronné, sans passer par la réécriture.

Un texte ne se construit pas sans prise en compte du lecteur capable de l'actualiser. Le texte représente une manifestation verbale qui permet au lecteur d'entrer dans le labyrinthe de l'implicite pour arriver à saisir du sens. Le lecteur appréhende le texte en fonction de ses expériences littéraires et culturelles. Plus les connaissances acquises par le destinataire sont larges, plus le texte latent prend de l'envergure: la formation du texte sous-jacent est en corrélation avec le savoir encyclopédique du lecteur. S'il en est ainsi, comment est-il possible que le lecteur enfant et le lecteur adulte soient impliqués dans un seul et même texte?

Lorsqu'il envisage le «lecteur modèle», Eco (1985: 37) définit deux souscatégories: le «lecteur modèle naïf» et le «lecteur modèle critique». Le lecteur modèle est une figure virtuelle qui répondrait parfaitement aux sollicitations d'un texte donné: il représente l'ensemble des compétences requises pour actualiser le texte (Eco 1985: 71, 77). Ce lecteur modèle se dédouble: d'un côté, le lecteur modèle naïf effectue une lecture sémantique et se laisse fasciner par le monde fictif et prendre aux pièges que l'auteur lui tend; de l'autre, le lecteur modèle critique appréhende la complexité de la structure textuelle. Le texte peut ainsi prendre en compte le double lecteur modèle selon l'ampleur des connaissances encyclopédiques de chacun (Eco 2007 : 261). Aussi le double lectorat (enfant/adulte) peut-il constituer une illustration concrète du double lecteur modèle. Il est à noter que ces deux niveaux de lecture ne présentent pas d'infériorité ou de supériorité l'un par rapport à l'autre. La lecture sémantique n'est pas moins importante que la lecture critique, car le texte prévoit son double sort interprétatif de cette façon.

Dans le texte impliquant un double lectorat, certaines parties se prêtent à la double réception, alors que d'autres ne peuvent donner une piste d'interprétation qu'au lecteur adulte. Une telle réception asymétrique peut résulter par excellence de l'intertextualité (Eco 2007: 261), car un lecteur moins cultivé court en effet le risque de ne pas saisir les valeurs de répétition de l'intertextualité. Celle-ci a d'abord été réservée au domaine littéraire pour démontrer que la production et l'interprétation d'un texte ne sont pas seulement une affaire intratextuelle, mais concernent également la mise en relation d'un texte donné et de textes antérieurs (Kristeva 1969). Désormais, l'intertextualité est considérée comme touchant d'autres formes d'expression artistique ou culturelle (Allen 2000 : 174). Étant donné que notre étude a pour objet d'analyse les albums qualifiés d'iconotextes, où coexistent le verbal et le visuel, l'intertextualité est ici comprise au sens large: elle implique une relation non seulement littéraire, mais aussi extralittéraire. 
Qu'elle soit littéraire ou non, l'intertextualité demande au lecteur une coopération active pour identifier la présence et la raison d'être de fragments sémiotiques préexistants dans un texte donné. Aussi l'intertextualité crée-t-elle une relation d'inclusion ou d'exclusion selon que le lecteur est enfant ou adulte. Si un réseau intertextuel est reconnaissable par les deux groupes de lecteurs, il constitue une relation d'inclusion en ce sens qu'il embrasse les deux. En revanche, si le réseau n'est identifiable que par l'un d'entre eux, il exclut l'autre. Vu que le repérage des intertextes dépend de la culture et de la sagacité du lecteur, l'enfant court davantage le risque d'être écarté du réseau intertextuel que l'adulte. Toutefois, le lecteur naïf n'est pas complètement exclu du réseau sémantique. Il peut arriver à construire le sens d'un fragment sémiotique sans tenir compte de l'hypotexte dont il fait originairement partie. Il s'agit d'une appréhension du fragment sémiotique dans son contexte immédiat (intratextuel), et non pas dans son contexte «indirect» (intertextuel). Le lecteur chevronné est quant à lui capable d'envisager le fragment à la fois dans ses contextes immédiat et indirect. On peut schématiser cela comme suit:

FIGURE 1

Intertextualité et rapport d'inclusion ou d'exclusion

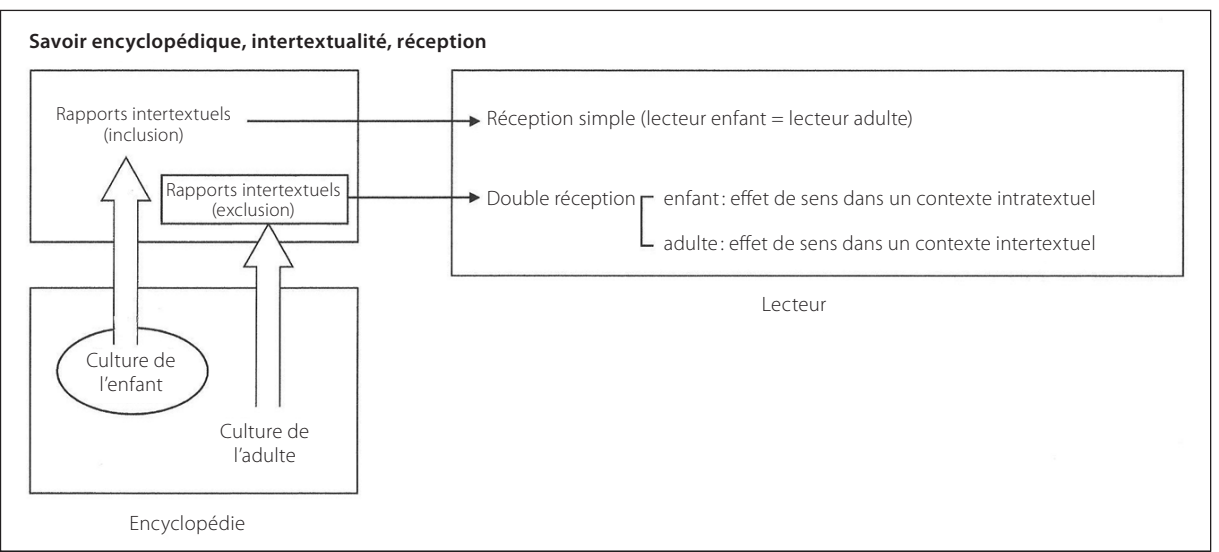

Si l'intertextualité repose sur la culture de l'enfant, elle peut être repérée par les deux lectorats; en revanche, si elle concerne la culture de l'adulte, le lecteur enfant essaie malgré tout de construire du sens en dehors de la chaîne intertextuelle.

Le réseau intertextuel, qui entraîne un double niveau de lecture, s'insère verbalement ou visuellement dans un album et peut avoir son origine dans le patrimoine littéraire, l'héritage artistique, voire la culture de masse. Cependant, la mise en relation du double lectorat et des albums est à première vue difficile à concevoir. On réserve plutôt les albums au lecteur enfant et on est enclin à les classer par tranches d'âge. Cette tendance est diamétralement opposée à la notion de double lectorat, qui justement refuse la correspondance entre un texte et un lectorat défini par l'âge. Les livres d'images sont en réalité caractérisés par leur appartenance à un double monde: la réception des albums concerne tant l'adulte que l'enfant. L'adulte est un médiateur reliant l'enfant au texte. Les albums font souvent l'objet d'une lecture accompagnée ou d'une lecture à haute voix, car le lecteur originairement visé - l'enfant - n'est pas encore très habitué à lire. 
Cependant les albums contemporains font de l'adulte non seulement un médiateur, mais aussi un véritable lecteur. Ils ont une forte tendance à viser le lecteur adulte, sans pour autant négliger leur lecteur traditionnel, comme le fait remarquer Beckett:

Perhaps more than any other genre, the picturebook has redrawn boundaries and expanded literary horizons in recent years. Contemporary picturebook makers continually are breaking new ground and challenging accepted forms and conventions. Although the picturebook traditionally has been seen as a children's genre, in the eyes of many contemporary authors and illustrators, it is a narrative form that can address any or all age groups. (Beckett 1999: xvi)

On assiste à une multiplication des livres d'images incitant l'adulte à devenir un lecteur pour son propre plaisir esthétique. Un tel élargissement du lectorat résulte de l'évolution de la perception de ce genre verbovisuel: F. Ruy-Vidal affirmait dans les années 1970 qu'«il n'y a pas d'art pour l'enfant, il y a de l'art. Il n'y a pas de graphisme pour enfants, il y a le graphisme. Il n'y a pas de couleurs pour enfants, il y a les couleurs. Il n'y a pas de littérature pour enfants, il y a la littérature ${ }^{5}$ ». Influencés par cette perspective avant-gardiste, les albums contemporains exploitent les patrimoines littéraires, artistiques et culturels pour susciter la curiosité du lecteur, surtout adulte. La reconnaissance et le déchiffrage d'un réseau intertextuel sont en corrélation avec l'étendue des connaissances encyclopédiques du lecteur, mais l'intertextualité n'est pas toujours réservée au lecteur cultivé. En effet, certaines références artistiques, littéraires et culturelles, insérées dans les albums, font partie de la culture des enfants, tandis que d'autres appartiennent plutôt au monde des adultes. L'intertextualité basée sur le bagage de l'enfant est accessible au double lectorat et n'entraîne pas une double réception; en revanche, le réseau intertextuel, associé à la culture adulte, est perceptible selon le degré de culture du lecteur.

\section{Traitement de l'intertextualité dans la traduction des albums}

La question «Comment faut-il traduire?» est inséparable de la question «Quelle est la visée de la traduction?» L'objectif peut définir les stratégies pour y parvenir. Comment traduire les albums destinés à un double lectorat? Le texte littéraire destiné au double lectorat tire son origine de la littérature jeunesse, mais se prête à une lecture plurielle. Le lecteur est considéré comme un agent essentiel en littérature de jeunesse, ainsi que l'affirme Jouve (2002: 73): «le destinataire des récits pour enfants [...] est [...] sensiblement différent du destinataire des récits pour adultes.» La littérature pour la jeunesse (dont les albums) mettant en relief la réception, le destinataire constitue un paramètre important pour sa traduction (Oittinen 2000; O'Sullivan 2005).

Les albums destinés à un double lectorat sont caractérisés par une double réception: c'est toujours le lecteur qui est en jeu. Par rapport à l'intertextualité, le lecteur est porteur d'un ensemble de patrimoines littéraires, artistiques et culturels qu'il s'est approprié dans une culture déterminée avant de rencontrer un texte donné; et il dispose d'une aptitude à en mobiliser des éléments pertinents pour repérer le réseau intertextuel inséré dans un texte. L'effet de lecture varie donc selon que le lecteur est enfant ou adulte. Dans cette perspective, il est possible de dire que la traduction de l'intertextualité insérée dans un album consiste à reconstruire un réseau sémiotique qui puisse produire des effets de lecture analogues chez le lecteur du texte produit. Nous allons désormais analyser la traduction de rapports intertextuels relevés dans 
des albums en trois langues - anglais, coréen et français. Par commodité, l'intertextualité est présentée selon le genre de l'hypotexte: beaux-arts, littérature et culture de masse.

\subsection{Intertextualité liée aux arts plastiques}

Lalbum étant un texte graphique-littéraire, la référence artistique est courante pour engendrer une double lecture (Beckett 2011b : 147). Le recyclage d'un hypotexte graphique dans un album est appelé "intertextualité visuelle» ou "intericonicité» (Nières-Chevrel 2008: 89; Renaud 2007: 190). Willy the Dreamer de Browne (1997) ${ }^{6}$ témoigne d'une exploitation riche et ludique du patrimoine artistique. Lauteurillustrateur puise dans l'univers artistique des surréalistes, entre autres Magritte et Dali. Dans cet iconotexte, chaque page montre ce que le héros, Willy, rêve de devenir : elle abrite un texte très simple et une illustration pleine de rapports intertextuels. À la différence du texte, les illustrations de l'album sont assez complexes pour rendre possible une lecture plurielle.

\section{IMAGE 1}

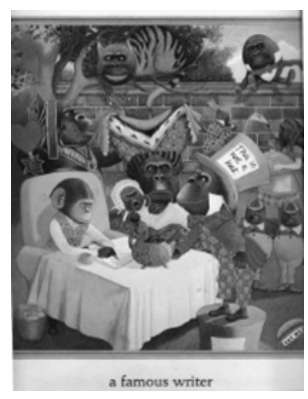

Copyright (c) 1997 A.E.t. Browne \& Partners

Dans l'image 1, le texte dit simplement que Willy veut devenir un écrivain célèbre, tandis que l'illustration permet de deviner qui est l'écrivain modèle de ce jeune héros. Les gorilles, personnages presque omniprésents dans le monde imaginaire de Browne - lesquels témoignent de la grande admiration de l'auteur pour le film américain King Kong - évoquent ceux des œuvres de Lewis Carroll: la Reine de Cœur, le Valet de Cœur, le Chapelier fou, le Chat de Cheshire, Tweedle Dee et Tweedle Dum. Derrière cette image se dissimulent trois textes virtuels: des illustrations de Tenniel, l'illustrateur le plus reconnu des œuvres de Carroll (images 2, 3 et 4). Ce réseau intertextuel permet à l'illustration de coopérer avec le texte pour préciser que le héros veut devenir un auteur aussi reconnu que Carroll.

En plus des illustrations de Tenniel, une œuvre d'art est exploitée dans cette page pour permettre une double réception. Sur le chapeau jaune du lapin, est inscrit «This is not a hat». Cette incrustation du verbal dans le visuel est qualifiée d'intraiconic text (texte intra-iconique) (Nikolajeva et Scott 2001: 118); elle sert à renforcer la dynamique iconotextuelle afin d'influer sur la réception de la partie en question.

D'après l'illustration - [image4] - de Tenniel, le chapeau jaune aurait dû contenir une autre expression: "In this style 10/6» (Dans ce style 10/6). Mais cette expression 
IMAGE 2

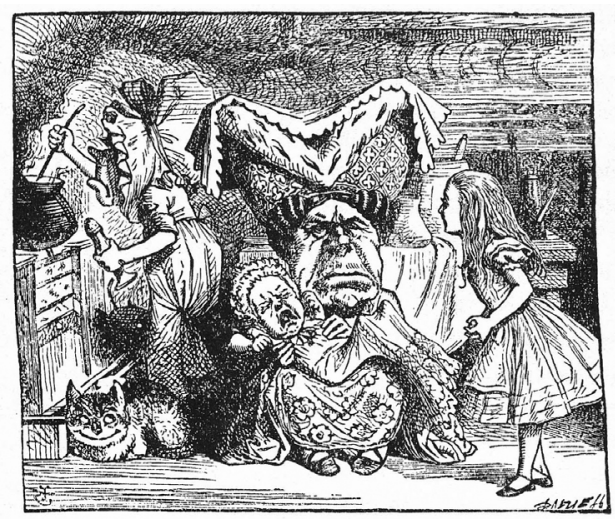

IMAGE 3

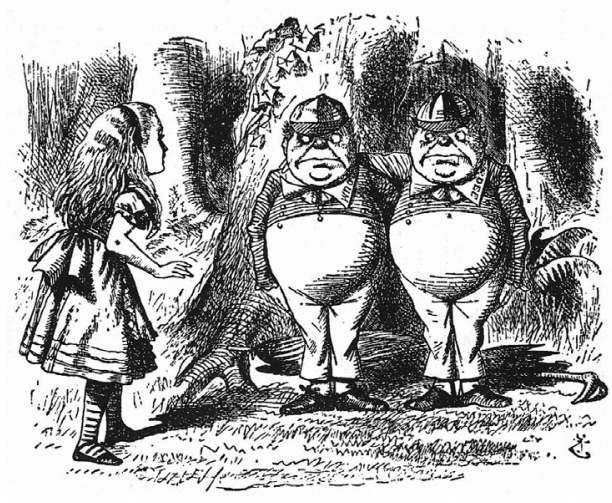

IMAGE 4

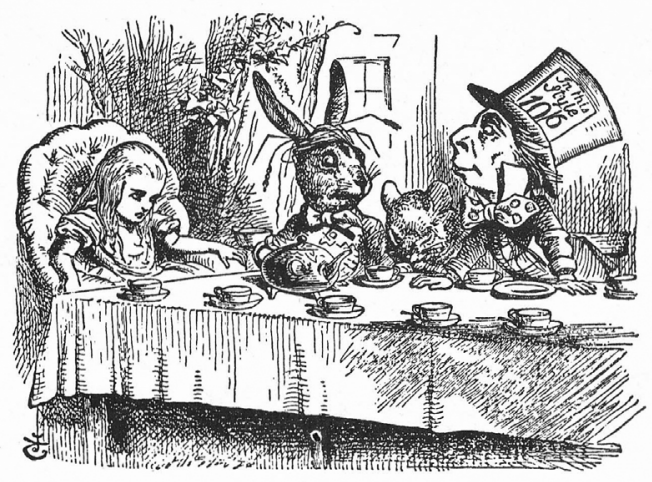

est remplacée par «This is not a hat», une parodie de l'œuvre de Magritte, La Trahison des images (Ceci n'est pas une pipe). Ce petit fragment textuel est interprétable à deux niveaux selon l'ampleur du savoir du récepteur. Le lecteur cultivé est capable de reconnaître l'hypotexte visuel et d'apercevoir le jeu parodique associé. C'est le contexte «indirect» qui fait surgir cet effet de lecture. Il faut reconnaître l'intertextualité en chaîne (l'expression dans l'illustration de Tenniel, puis celle dans le tableau de Magritte) pour parvenir à cette lecture approfondie. Le lecteur enfant, qui n'a pas encore cette œuvre d'art dans son bagage, risque de ne pas établir le lien intericonique, mais il peut effectuer une autre lecture, hédoniste. L'album étant un espace intersémiotique, le rapport verbovisuel y est essentiel pour produire un effet de lecture. En général, on en distingue trois types: la redondance, la collaboration et la disjonction (Van der Linden 2006: 120-121). Il y a redondance quand le texte et les illustrations montrent une même histoire; collaboration quand les deux se relaient pour développer la narration; et disjonction quand le textuel et le visuel se contredisent. L'image du chapeau jaune et l'expression verbale produisent une ironie flagrante: le visuel montre un chapeau, alors que le verbal dit que ce n'en est pas un. Ce lien intersémiotique produit un effet ludique sur le lecteur enfant sans exiger qu'il 
mobilise du savoir encyclopédique. Il s'agit d'un effet de lecture surgissant du contexte immédiat, sans rapport avec la référence artistique. Bien que cette intertextualité ne soit pas accessible au lecteur enfant, une autre lecture est possible, tout aussi cohérente, plus immédiate, sans recours au bagage culturel du lecteur. L'écart entre la culture de l'enfant et celle de l'adulte permet une double actualisation textuelle. L'une est aussi légitime que l'autre.

L'expression intra-iconique de l'album de Browne a été traduite en français et en coréen de la façon suivante:

(1) Ceci n'est pas un chapeau (traduit par Isabel Finkenstaedt) ${ }^{7}$

(2) 이것은 모자가 아니다! (traduit par Ji-wone Lee) ${ }^{8}$

[Ceci n'est pas un chapeau!]

Le fragment recyclé ne peut entraîner une lecture intertextuelle en traduction que s'il fait partie de la mémoire du lecteur de la culture réceptrice. Le tableau de Magritte peut servir d'hypotexte aussi bien dans les cultures française et coréenne que dans la britannique. Le tableau La Trahison des images de l'artiste belge est une œuvre familière aux Coréens. La traduction française consiste en un retour sans faute à l'expression originale qui appartient au patrimoine artistique français, en vue d'assurer une éventuelle double lecture associée à ce facteur extralittéraire. La traduction française reste fidèle à l'expression de Magritte gravée dans la mémoire du lectorat, excepté le remplacement de «une pipe» par «un chapeau». La valeur de répétition permet au lecteur cultivé de rétablir le rapport entre le texte intra-iconique et l'œuvre de Magritte dans la culture de réception. Il n'existe pas d'écart interculturel entre la culture de réception et celle d'origine. L'écart intergénérationnel demeure important dans la culture de réception. L'intertextualité en question concerne toujours le lectorat adulte, à l'exclusion du lectorat enfant. De ce fait, la traduction française reste linguistique et culturellement fidèle à l'original.

La traduction coréenne présente quant à elle une légère différence par rapport au texte original et à la traduction française: elle se termine par un point d'exclamation. La ponctuation ne relève pas seulement de normes d'écriture, mais peut également participer à l'effet de sens (Rosier 1998: 21). Le point d'exclamation qui clôt la traduction coréenne constitue un ajout qui affecte la réception. Il accentue le contraste entre l'image du chapeau et l'expression intra-iconique, en dénaturant quelque peu l'intertextualité, et renforce l'effet de lecture produit sur le lecteur enfant. Ce choix traductif privilégie le contexte immédiat - le rapport verbovisuel - et le lecteur enfant, en atténuant le lien intertextuel - le contexte indirect - et la curiosité du lecteur adulte.

\subsection{Intertextualité littéraire}

Si l'hypotexte n'est accessible qu'au lecteur adulte, le rapport intertextuel constitue un jeu littéraire «repérable» pour le lecteur adulte. Cette intertextualité à caractère exclusif conduit le lecteur adulte vers l'intertexte dont fait partie la référence, alors que le lecteur enfant fait une lecture linéaire (Gignoux 2005: 38). En revanche, lorsque l'hypotexte appartient à la fois à la sphère culturelle de l'enfant et à celle de l'adulte, l'intertextualité entraîne pour tous deux une lecture en aller-retour entre l'hypertexte et l'hypotexte. 
L'album de Colin Thompson, How To Live Forever $(1995)^{9}$, semble poser la question du syndrome de Peter Pan (1911) ${ }^{10}$. Il s'agit d'un retournement du roman écossais de Barrie. C'est l'histoire d'un garçon vivant avec sa famille dans une bibliothèque magique: «Toutes les nuits, il part à la recherche d'un livre disparu, ce qui le mènera au secret qui permet de rester éternellement enfant.» Puisque le lieu du récit est une bibliothèque et que «le livre disparu» est au centre de l'histoire, les livres jouent un rôle essentiel dans cet iconotexte en constituant un espace où se tisse un grand réseau intertextuel. Une double page qui représente un rayonnage de bibliothèque plein de livres attire l'attention des lecteurs, qui peuvent voir le titre de chaque livre placé sur les étagères.

\section{IMAGE 5}

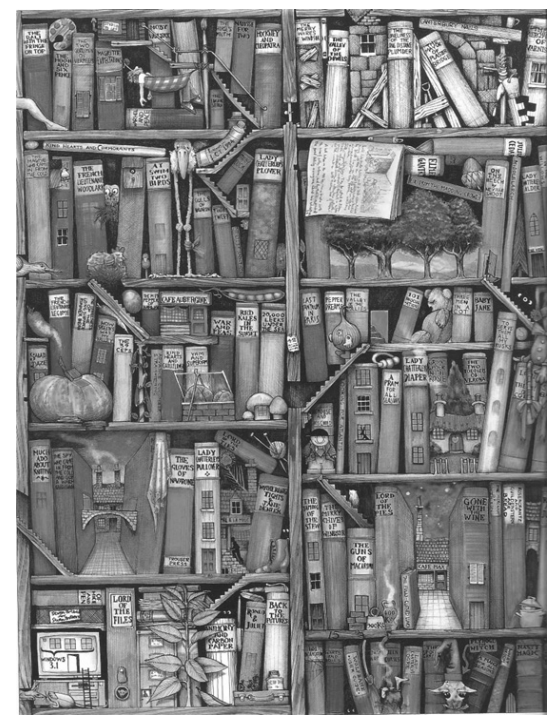

Copyright (C) 1995 Colin Thompson

Un titre fonctionne comme un nom propre pour désigner une œuvre et a un lien sémantique avec le contenu de celle-ci, en annonçant de quoi il parle. Le titre joue également un rôle accrocheur: il conduit le lecteur vers le texte, en programmant la lecture. Il arrive que le titre subsiste seul dans la mémoire après la lecture d'un livre. Il permet alors de construire un réseau intertextuel meilleur que tous les facteurs textuels: il représente et peut évoquer l'ensemble d'un texte. Ainsi tous les titres présents sur cette double page, qui appartiennent au patrimoine classique, se trouvent dans un rapport intertextuel.

Ces titres sont tous des parodies de titres originaux. En littérature, l'intertextualité se manifeste sous diverses formes: citation, allusion, pastiche, parodie, etc. Parmi elles, la parodie est fréquemment utilisée pour les textes littéraires destinés au double lectorat enfant-adulte, car la dérivation de textes antérieurs, qui garde un lien patent avec ceux-ci, a une visée ludique ou humoristique (Samoyault 2012: 37-38). Si le lecteur est capable de relier les titres parodiques à leur forme originale, cette double page devient pour lui un espace de jeux de mots basés sur l'intertextualité. Les titres, bien qu'insérés dans l'image, sont tous traduits dans la version française et dans la 
version coréenne, afin de préserver l'effet de lecture qui dépend du savoir préalable du lecteur.

Certains titres font partie de la littérature jeunesse et d'autres n'appartiennent qu'à la littérature pour "adulte». Les premiers sont donc reconnaissables par les enfants et les adultes, alors que les seconds ne sont repérables que par ces derniers. Cela étant, les titres entraînent une relation d'inclusion ou d'exclusion selon le bagage du lecteur, et ont pour effet une double lecture. À titre d'illustration, Lady Chatterley's Plover est une parodie de Lady Chatterley's Lover de D. H. Lawrence ${ }^{11}$, qui est indéniablement une œuvre pour adultes. En revanche, The Lizard of $\mathrm{O} z$ est une dérivation de The Wonderful Wizard of $\mathrm{O} z$ de L. F. Baum ${ }^{12}$, un roman pour la jeunesse.

À propos du double lectorat, la traduction française et la traduction coréenne présentent des tendances différentes. Si l'on prend Lady Chatterley's Plover comme exemple, la version française donne Ali Baba et les 40 Rockers, qui dérive d'Ali Baba et les 40 voleurs. Il s'agit de jeux de mots intertextuels. Pour Lady Chatterley's Plover, «lover» du titre original devient «plover». C'est une parodie intertextuelle qui n'est reconnaissable que par le lecteur adulte. Le lecteur enfant, lui, peut faire une lecture iconotextuelle de cette expression dans son contexte immédiat. La façon de parodier les titres dans cet album est associée aux illustrations: on voit plusieurs espèces d'oiseau dans le compartiment de la bibliothèque où se trouve Lady Chatterley's Plover.

\section{IMAGE 6}

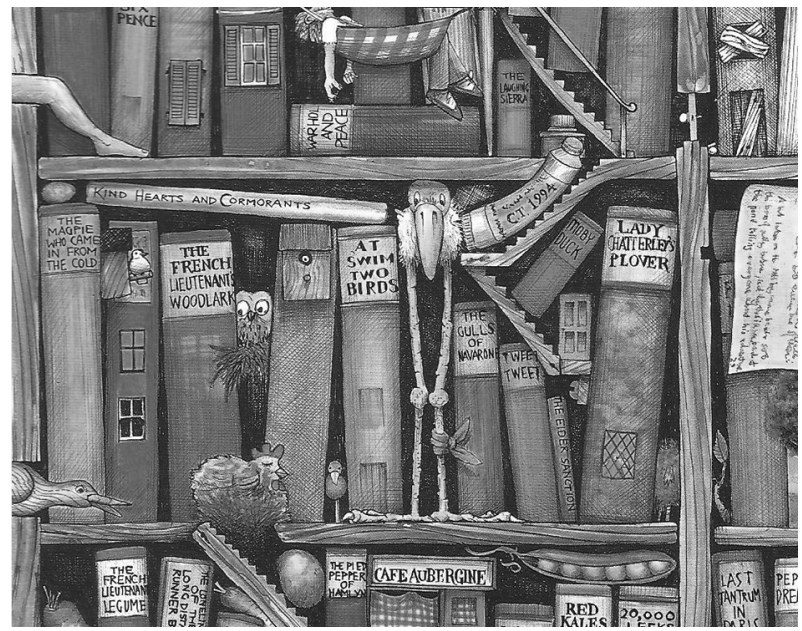

Au dos de ce livre, il y a une image d'un petit oiseau derrière une grille. Le lecteur enfant, bien qu'exclu du réseau intertextuel, peut faire une lecture intersémiotique, en associant le titre parodique à l'image.

La version française ${ }^{13}$ transfère le jeu de mots, mais sans maintenir l'écart intergénérationnel, car Ali Baba et les 40 voleurs fait partie du patrimoine littéraire pour enfants. Bien que l'œuvre Lady Chatterley's Lover soit bien intégrée à la culture française (au point qu'il en a été fait un film français), la version française de l'album de Thompson abandonne l'intertextualité basée sur un roman pour adultes pour adopter le réseau intertextuel identifiable par le lecteur enfant. Elle transforme alors la double réception en réception simple. 
La version coréenne ${ }^{14}$, elle, fait passer le jeu de mots intertextuel sans négliger la double réception. Lady Chatterley's Plover devient «채털리 부인의 사냥 [La chasse de Lady Chatterley] »: on retrouve le jeu de mots au même endroit. Le titre coréen du roman pour adultes est «채털리 부인의 사랑 [Tchae-teol-ri Bu-in-ui Sa-rang)]», et le dernier mot, «sa-rang» [사랑, amour] devient «sa-nyang» (사냥, chasse) pour former le titre coréen parodique. Il y a bien ici un jeu phonétique avec un changement de lettres, tout comme entre «lover» et "plover». La version coréenne fait non seulement un jeu de mots intertextuel, mais vise aussi le double lectorat. Le lecteur adulte coréen repère le jeu lié à une œuvre classique, alors que le lecteur enfant est exclu du réseau intertextuel et ne comprend le titre en question que dans son contexte immédiat, sans reconnaître l'effet de parodie. Les titres coréens parodiques du même compartiment sont tous liés aux oiseaux, mais pour celui que nous commentons, «채털리 부인의 사냥 [la chasse de lady Chatterley]», il s'agit de «la chasse», une activité de forêt - la chasse aux oiseaux - pour transférer le jeu phonétique. Alors ce choix n'est pas sans rapport à l'image du dos de Lady Chatterley's Plover.

Lady Chatterley's Lover est parodié d'une autre manière dans un autre compartiment de la bibliothèque: il s'agit de Lady Chatterley's Pullover avec l'ajout de "pul» à «lover». Encore un jeu de mots intertextuel, dont l'hypotexte n'est reconnaissable que par le lecteur adulte. Le lecteur enfant peut quant à lui appréhender ce titre parodique en fonction de l'illustration: le compartiment de la bibliothèque où se trouve le livre est visuellement réservé à l'habillement: écharpe, botte, fils et aiguilles à tricoter, plumes de paon, etc.

\section{IMAGE 7}

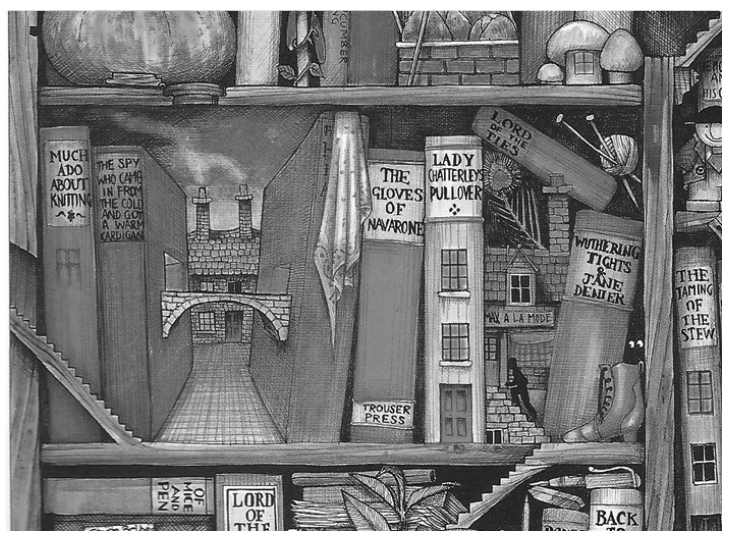

La version coréenne «채털리 부인의 오버 [le manteau de Lady Chatterley]» présente un jeu de mots qui maintient aussi le réseau intertextuel accessible seulement par le lecteur adulte. Dans la version française, le titre Les musiciens de la fille de Brême dérive des Musiciens de la ville de Brême. Il est évident que le jeu de mots tient compte de l'intertextualité. Toutefois, l'hypotexte concerné fait partie du patrimoine littéraire pour la jeunesse: c'est une œuvre des frères Grimm qui remplace le roman de Lawrence. Ainsi le réseau intertextuel dans la version française constitue une relation «inclusive» du double lectorat.

Le texte original contient non seulement un réseau intertextuel «exclusif», mais aussi un réseau «inclusif». À titre d'illustration, 20000 Leeks Under The Sea » a pour 
hypotexte Twenty Thousand Leagues Under The Sea de Jules Vernes, œuvre française plutôt destinée à la jeunesse, et The Lizard of $\mathrm{O} z$ » est une parodie de The Wonderful Wizard of $\mathrm{O} z$, classique américain pour la jeunesse. Cependant, parmi les titres parodiques, ceux basés sur le patrimoine littéraire adulte sont beaucoup plus nombreux que ceux liés à la littérature pour la jeunesse. Ainsi, face à cette double page, le lecteur adulte s'appuie sur le contexte «distant» en mobilisant ses expériences littéraires précédentes, alors que le lecteur enfant appréhende la majorité des titres parodiques dans leur contexte immédiat. La relation d'exclusion est donc plus importante que celle d'inclusion. Cependant, la version française présente une proportion entre littérature pour adulte et littérature pour la jeunesse différente de l'original. Le réseau intertextuel «inclusif» est prépondérant par rapport au réseau «exclusif» (voir la figure 2 ci-dessous). Les titres français visent moins la double réception et incluent plus souvent le jeune lectorat dans un réseau intertextuel. Dans la version coréenne se retrouve la même proportion entre titres pour adultes et pour enfants que dans le texte original. La corrélation entre la double réception et l'intertextualité y est préservée (voir l'annexe 1).

\subsection{Intertextualité liée aux références culturelles}

L'album français Idora d'Alex Godard $(1997)^{15}$ semble destiné aux enfants en bas âge: il a pour personnages des animaux anthropomorphiques, comme dans les fables, et son histoire est assez facile à suivre. Cet iconotexte qui paraît si simple ne parle pourtant pas de thèmes très légers: il s'agit, d'une part, de la quête du bonheur sur le plan individuel; d'autre part, sur le plan social, de la démolition d'un immeuble et du départ forcé des résidents. De plus, l'album dépasse la culture enfantine par endroits avec quelques expressions qui donnent un plaisir inattendu au médiateur adulte lisant à haute voix à l'enfant. Tout en restant fidèles à leur jeune lectorat (le lectorat primaire), les auteurs pour la jeunesse emploient parfois certaines expressions pour faire un clin d'œil au lectorat adulte (le lectorat secondaire).

Dans Idora, un nom de personnage vise le double lectorat: la girafe Idora a une voisine éléphante qui s'appelle Bossanova. Ce nom est lié au métier de l'éléphante qui est chanteuse, la «bossa-nova» étant un style musical brésilien. La reconnaissance de la référence culturelle, c'est-à-dire la mise en relation entre le textuel et l'extratextuel, concerne le lecteur adulte plutôt que le lecteur enfant, car le genre musical évoqué fait partie de la culture de l'adulte plus que de l'enfant. Ce dernier est donc exclu du réseau intertextuel. Cependant, le nom enchante l'enfant d'une

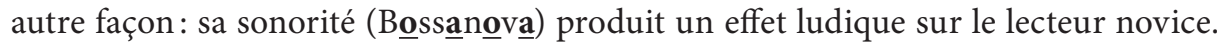
Bien qu'il soit écarté de la relation intertextuelle, il peut se plaire au jeu phonétique impliqué dans ce nom, surtout en écoutant la lecture à haute voix. Les jeux phonétiques sont l'une des caractéristiques de la littérature de jeunesse. La sonorité est d'autant plus importante dans les textes littéraires pour enfants que ceux-ci requièrent un médiateur adulte pour leur faire la lecture. Les enfants lisent donc les albums avec les oreilles. À titre d'exemple, l'auteur-illustrateur français Philippe Corentin ${ }^{16}$ donne «Pipioli» comme nom à l'un de ses héros; le sens du nom se perçoit plus avec les oreilles qu'avec les yeux. De même, dans «Bossanova », la répétition des voyelles [o] et [a] constitue une sonorité pouvant amuser l'enfant. Ainsi, chaque lecteur trouve son compte dans ce nom: le lecteur cultivé appréhende «Bossanova» 
dans son contexte musical, alors que le lecteur naïf y trouve du plaisir dans son contexte immédiat.

Dans la version coréenne ${ }^{17}$, «Madame Bossanova» est devenue «코끼리 아줌마 [ko-ggi-ri a-jum-ma] (Madame Éléphant)», ce qui n’est pas la transcription phonétique du nom original et n'évoque pas non plus la référence culturelle. Sur quoi une telle recréation est-elle fondée? C'est dans l'image que se trouve la réponse. Cette adaptation ponctuelle - selon le terme de Bastin (1993: 473-478) - est fondée sur l'illustration.

FIGURE 2

\section{Changement d'hypotexte}

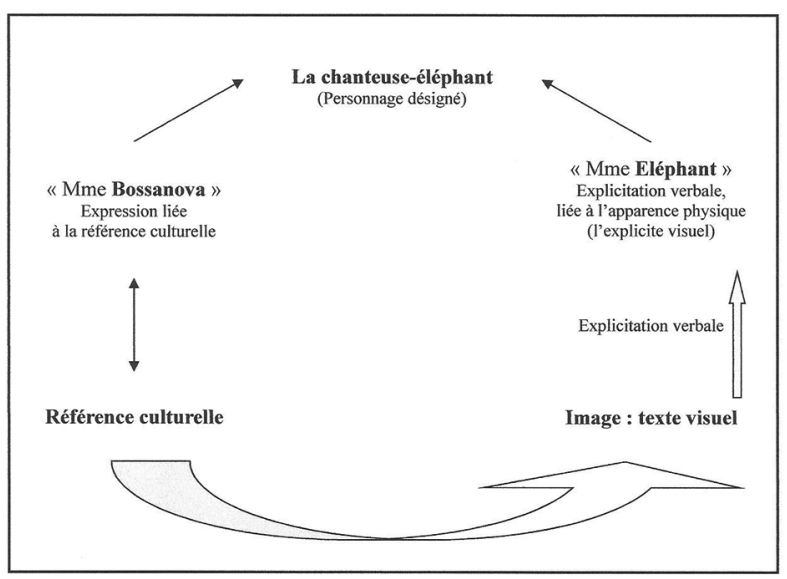

La corrélation entre «Madame Bossanova» et sa référence culturelle est remplacée par la relation motivée entre un nouveau nom «Madame Éléphant» et son portrait graphique. Autrement dit, le texte visuel permet au traducteur de donner à la chanteuse-éléphant un nouveau nom, plus familier au lecteur enfant du texte d'arrivée. Ce choix semble prendre en compte l'affinité entre l'animal anthropomorphe et l'enfant (Chelebourg et Marcoin 2007: 97).

Cependant «Bossanova» peut être phonétiquement transcrit en écriture coréenne et «Madame» peut être traduit par son équivalent coréen, de sorte que «Madame Bossanova» peut être rendu par «보사노바 아줌마 [bo-sa-no-va a-jum-ma]»«아줌마» est une appellation coréenne pour les femmes mariées. Le nom de ce style musical brésilien ayant déjà été emprunté tel quel par la culture coréenne, la transcription phonétique du nom propre permettrait de préserver le double niveau de lecture. Si le lecteur enfant de l'original, de même que celui de la traduction, ne reconnaît pas la référence culturelle liée au nom, cela signifie que la non-reconnaissance de la référence culturelle par le lecteur enfant est un effet voulu par le texte original. En rompant avec le réseau intertextuel, la traduction neutralise l'effet de double lecture. La relation d'exclusion devient celle d'inclusion sur le plan de la réception.

Le traducteur de la version anglaise ${ }^{18}$ a fait un choix différent: il a conservé le nom original. La bossa-nova est un genre musical bien présent dans la culture angloaméricaine, et cette appellation brésilienne y est employée telle quelle. Le nom de l'éléphante peut entraîner une double lecture selon la reconnaissance du réseau 
intertextuel dans la culture de réception. Cette solution préserve l'écart entre la culture des adultes et celle des enfants, et donc la relation d'exclusion.

\section{En guise de conclusion}

Le texte littéraire pour la jeunesse peut viser un double lectorat à travers des rapports intertextuels. Les hypotextes font partie soit de la culture des enfants, soit de celle des adultes : les premiers entraînent une relation d'inclusion, car ils touchent les deux lectorats, alors que les seconds induisent une relation d'exclusion, puisqu'ils écartent le jeune lecteur du réseau intertextuel - en lui permettant toutefois d'appréhender les expressions dans leur contexte immédiat. Face à ce genre de texte, la traduction peut préserver la relation d'exclusion du texte de départ ou la convertir en une relation d'inclusion. Le premier choix permet au texte d'arrivée de jouer du double niveau de lecture et d'offrir un feuilleté sémantique, tandis que le second réduit la potentialité de jeux sémantiques et conduit à une lecture sans écart entre les deux lectorats.

FIGURE 3

Réception simple et double réception dans la traduction

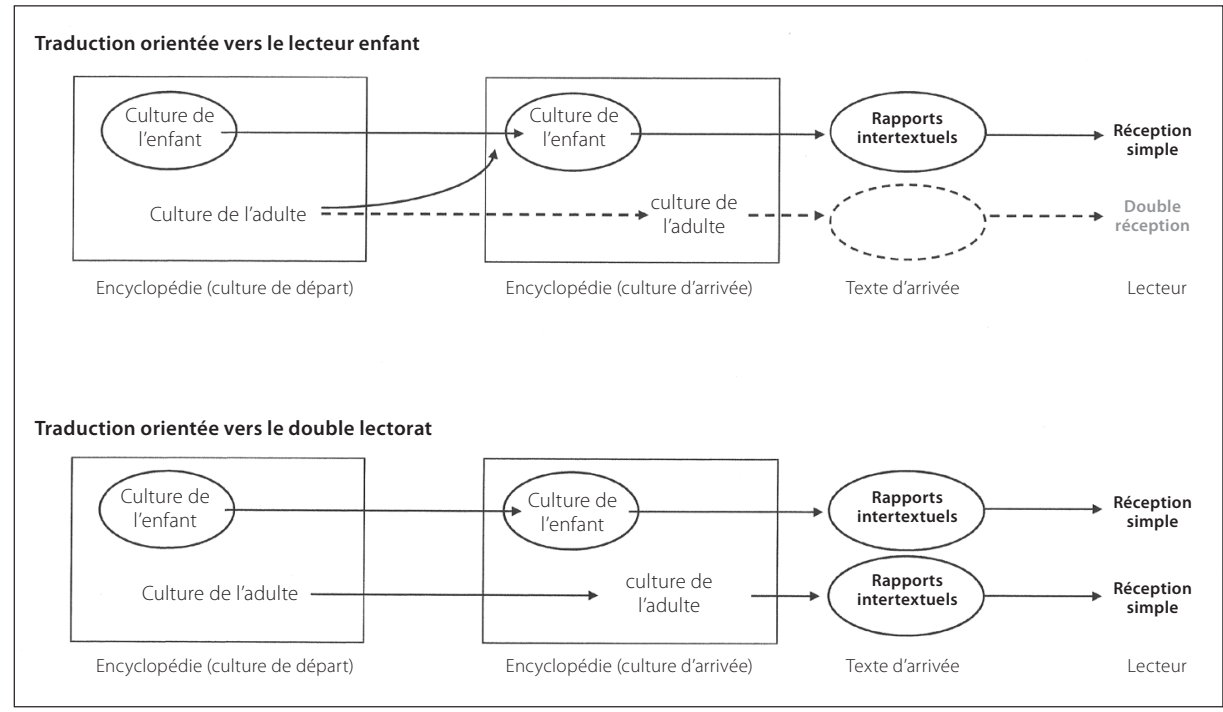

Comme le montre le schéma 3, la traduction est orientée vers la réception simple si la culture de l'adulte, dont fait partie le réseau intertextuel, est remplacée par la culture de l'enfant. En revanche, le texte d'arrivée peut entraîner la double réception, quand l'hypotexte en question appartient à la culture de l'adulte comme dans le texte de départ. Les deux lectorats mobilisent respectivement le contexte immédiat et le contexte éloigné pour construire du sens.

Si la traduction tend à transférer le mécanisme intertextuel qui entraîne les deux niveaux de lecture, cela signifie qu'elle implique les quatre cultures suivantes: de départ et d'arrivée, de l'adulte et de l'enfant. Pour rétablir un réseau intertextuel dans le texte d'arrivée, il est essentiel d'évaluer l'hypotexte associé dans la culture d'arrivée. Si celui-ci a la même valeur de répétition dans la culture de réception que dans 
celle d'origine, sa relation intertextuelle, transférée dans le texte d'arrivée se prête à un jeu interprétatif analogue à celui du texte original. Si l'hypotexte lié au texte de départ n'a pas de valeur de répétition et de reconnaissance dans la culture de réception, le jeu intertextuel peut se produire par le remplacement d'un autre hypotexte qui puisse remplir la même fonction. Pour le double lectorat, la valeur de l'hypotexte ne varie pas seulement selon les cultures d'origine et de réception, mais également selon les cultures des enfants et des adultes. C'est la raison pour laquelle la traduction d'un texte de type «double lectorat» peut être définie comme un passage entre deux langues et entre quatre cultures ou quatre encyclopédies. Pour le texte à effet de double lecture, l'interculturel est associé non seulement à l'interlinguistique, mais aussi à l'intergénérationnel.

\section{NOTES}

1. Perrault, Charles (1967): Les Contes de la mère l'Oye ou Histoires et contes du temps passé. Paris: Édition Claude Barbin.

2. SwIFT, Jonathan (1726): Gulliver's Travels (Voyages de Gulliver en français). Londres: Benjamin Motte.

3. CARroll, Lewis (1865): Alice's Adventures in Wonderland (Alice au pays des merveilles en français). Londres: Macmillan and Co.

4. Tournier, Michel (1971): Vendredi ou la Vie sauvage. Paris: Gallimard.

5. Le site du Centre National de la Littérature de jeunesse La Joie par les Livres (dernière mise à jour du site le 2 juillet 2008). Consultée le 28 décembre 2013. <lajoieparleslivres.bnf.fr/masc/Integration/ JOIE/statique/univ/interfaceschoisies/Ruy-Vidal/rubrique_edition_acteur_bio.html>.

6. Browne, Anthony (1997): Willy the Dreamer. Londres: Walker Books.

7. Browne, Anthony (1997): Marcel le rêveur. (Traduit par Isabel Finkenstaedt). Paris: Kaléidoscope.

8. Browne, Anthony (1997/2004): 꿈꾸는 윌리 [Willy qui rêve]. (Traduit par Eun-Mi HuH). Séoul: Woongjin.

9. Thompson, Colin (1995): How To Live Forever. Londres: Random House.

10. Berrie, James Matthew (1911): Peter and Wendy. Londres: Hodder \& Stoughton.

11. Lawrence, David H. (1928): Lady Chatterly's Lover. Florence: Tipografia Giuntina.

12. Baum, L. Frank. (1900): The Wonderful Wizard of Oz. Chicago: George M. Hill Company.

13. Thompson, Colin (1995/1996): Le livre disparu. (Traduit par Catherine Bonнomme). Paris: Circonflexe.

14. Thompson, Colin (1995/2010): 영원히 사는 법 [Moyen pour avoir une vie éternelle]. (Traduit par Ji-wone LEE). Séoul: Nonjang.

15. GodArd, Alex (1997): Idora. Paris: Seuil Jeunesse.

16. Corentin, Philippe (1990): L’Afrique de Zigomar. Paris: L'École des Loisirs.

17. GodARD, Alex (1997/2001): 다 잘 될거야 [Tout ira bien]. (Nom du traducteur non indiqué). Séoul: Yejihyeon 2001.

18. GodArd, Alex (1997/1999): Idora. (Traduit par Laura McKenna). La Joella: Kane/Miller Book Publishers.

\section{RÉFÉRENCES}

Allen, Graham (2000): Intertextuality. London: Routledge.

BARThes, Roland (1968): La mort de l'auteur. Manteia. 5(4):12-17.

Bastin, Georges (1993): La notion d'adaptation en traduction. Meta. 38 (3):473-478.

Becкetт, Sandra Lee, dir. (1999): Transcending Boundaries. Writing for a Dual Audience of Children and Adults. New York: Garland Publishing.

Beckett, Sandra Lee (2011a): Crossover Literature. In: Philip Nel et Lissa Paul, dir. Keywords for Children's Literature. New York: New York University Press, 58-61.

Beckett, Sandra Lee (2011b): Crossover Picturebooks. A Genre for All Ages. Londres: Routledge. Chelelbourg, Christian et Marcoin, Francis (2007): La littérature de jeunesse. Paris: Armand Colin. 
Eco, Umberto (1985) : Lectorat in fabula ou la Coopération interprétative dans les textes narratifs (traduit par Myriem Bouzaher). Paris: Grasset.

Eco, Umberto (2007): Dire presque la même chose: Expériences de traduction (traduit par Myriem Bouzaher). Paris: Grasset.

Gignoux, Anne Claire (2005): Initiation à l'intertextualité. Paris: Ellipses.

IsER, Wolfgang (1985): L'Acte de lecture (traduit par Evelyne SznyCER), coll. «Philosophie et langage». Bruxelles: Éditions Mardaga.

Jauss, Hans Robert (1978): Pour une esthétique de la réception. Paris: Gallimard.

Jouve, Vincent (2002): Littérature pour adultes, littérature pour enfants: à chacun son jeu. La Revue des livres pour enfants. 206:69-77.

Kristeva, Julia (1969): Recherches pour une sémanalyse. Paris: Seuil.

KüMmerling-Meibauer, Bettina (1999): Crosswriting as a Criterion for Canonicity: the Case of Erich Kästner. In: Sandra L. Becketт, dir. Transcending Boundaries. Writing for a Dual Audience of Children and Adults. New York: Garland Publishing, 13-30.

LANSON, Gustave (1894) : Histoire de la littérature française. Paris: Hachette.

Nières-Chevrel, Isabelle (1984): L'adaptation dans les livres pour la jeunesse: lisibilité ou stratégie d'exclusion? Le français aujourd'hui. (68):80-85.

Nières-Chevrel, Isabelle (2008): Doubles images, doubles lecteurs: l'inter-iconicité dans Le Tunnel d'Anthony Browne. In: Christiane Connan-Pintado, Florence Gaiotti et Bernard Poulon. L'Album contemporain pour la jeunesse: nouvelles formes, nouveaux lecteurs? Modernités, $n^{\circ} 28$. Bordeaux: Presses Universitaires de Bordeaux, 89-99.

Nikolajeva, Maria et Scott, Carole (2001): How Picturebooks Work. New York: Garland Publishing.

Oittinen, Ritta (2000): Translating for Children. Londres: Garland Publishing.

O'Sullivan, Emer (2005): Comparative Children's Literature. Londres: Routledge.

Plassard, Freddie (2007): Lire pour traduire. Paris: Presses Sorbonne Nouvelle.

Renaud, Catherine (2007): Les «incroyabilicieux» mondes de Ponti. Une étude du double lectorat dans l'œuvre de Claude Ponti. Uppsala: Uppsala University.

Rosier, Laurence (1998) : La ponctuation et ses acteurs. In: Jean-Marc Defays, Laurence Rosier et Françoise Tilkin, dir. À qui appartient la ponctuation? Paris/Louvain-La-Neuve: De Boeck-Duculot. 15-30.

Samoyault, Tiphanie (2012): L'intertextualité - Mémoire de la littérature. Paris: Armand Colin. VAn der Linden, Sophie (2006): Lire l'album. Puy-en-Velay: L'Atelier du Poisson Soluble.

WALl, Barbara (1991): The Narrator's Voice: The Dilemma of Children's Fiction. New York: McMillan. 
ANNEXE 1

Comparaison entre l'original et la version française (hypotextes)

\begin{tabular}{|c|c|c|}
\hline & Hypotextes - Texte original (65) & Hypotextes - Traduction française (65) \\
\hline $\begin{array}{l}\text { Littérature } \\
\text { pour la } \\
\text { jeunesse }\end{array}$ & $\begin{array}{l}\text { The Pied Piper of Hamelin } \\
\text { Ali Baba and the Forty Thieves } \\
\text { Robinson Crusoe } \\
\text { Twenty Thousand Leagues Under the Sea } \\
\text { The Wind in the Willows }\end{array}$ & $\begin{array}{l}\text { Les Contes de Perrault } \\
\text { Le Livre de la jungle } \\
\text { Alice au pays des merveilles } \\
\text { Le Tour du monde en } 80 \text { jours } \\
\text { Ali Baba et les } 40 \text { voleurs } \\
\text { Le Comte de Monte-Cristo } \\
\text { Dix petits nègres } \\
\text { Le Chat perché } \\
\text { Le Chien des Baskerville } \\
\text { La Petite sirène } \\
\text { Histoire comme ça } \\
\text { Les Musiciens de Brême } \\
\text { Mes amis les loups } \\
\text { Le Pêcheur et sa femme } \\
\text { Les Voyages de Gulliver } \\
\text { Les Quatre filles du docteur March } \\
\text { La Guerre des boutons } \\
\text { Les Lettres de mon moulin } \\
\text { Arsène Lupin } \\
\text { Robinson Crusoé } \\
\text { Le Petit monde de Sophie } \\
\text { L'̂̂le au trésor } \\
\text { Vingt mille lieues sous les mers } \\
\text { Les Aventures de Tom Sawyer } \\
\text { Mémoires d'un âne } \\
\text { Un bon petit diable } \\
\text { Le Petit chaperon rouge } \\
\text { La Case de l'oncle Tom } \\
\text { La Petite fille aux allumettes } \\
\text { La Petite maison dans la prairie } \\
\text { La Princesse au petit pois } \\
\text { Les Trois mousquetaires } \\
\text { Le Vilain petit canard } \\
\text { On a marché sur la lune }\end{array}$ \\
\hline
\end{tabular}




\begin{tabular}{|c|c|c|}
\hline $\begin{array}{l}\text { Littérature } \\
\text { pour adultes }\end{array}$ & $\begin{array}{l}\text { The Surrey with the fringe on top } \\
\text { The Moon and sixpence } \\
\text { The Two Gentlemen of Verona } \\
\text { Great Expectations } \\
\text { War and Peace } \\
\text { Moby Dick } \\
\text { The Horse's Mouth } \\
\text { Three Men in a Boat } \\
\text { Antony and Cleopatra } \\
\text { Kind Hearts and Coronets } \\
\text { The Spy Who Came in from the Cold } \\
\text { The French Lieutenant's Woman } \\
\text { At Swim-Two-Birds } \\
\text { The Guns of Navaron } \\
\text { The Eiger Sanction } \\
\text { The Lady Chartterley's Lover } \\
\text { The Loneliness of the Long Distance } \\
\text { Runner } \\
\text { How Green was My Valley } \\
\text { The } 39 \text { Steps } \\
\text { Man and Superman } \\
\text { Much Ado about Nothing } \\
\text { Lord of the Flies } \\
\text { Wuthering Heights } \\
\text { Jane Eyre } \\
\text { Seven Brides for Seven Brothers } \\
\text { Of Mice and Men } \\
\text { Romeo and Juliet } \\
\text { Back to the Future } \\
\text { The Merry Wives of Windsor } \\
\text { The Valley of Ashes } \\
\text { The Mayor of Casterbridge } \\
\text { The Canterbury Tales } \\
\text { The Merchant of Venice } \\
\text { Elmer Gantry } \\
\text { Far From the Madding Crowd } \\
\text { On the Beach } \\
\text { Last Tango in Paris } \\
\text { Looking for Atlantis } \\
\text { A Man for All Seasons } \\
\text { To Serve Them All My Days } \\
\text { The Taming of the Shrew } \\
\text { Gone with the Wind } \\
\text { Rosencrantz and Guildenstern Are Dead } \\
\text { A Room with a View } \\
\text { Around the World in Eighty Days } \\
\text { The Hitchhiker's Guide to the Galaxy } \\
\text { Double, double toil and trouble } \\
\text { The Grapes of Wrath } \\
\text { King Rear } \\
\text { A Tale of Two Cities } \\
\text { Light a Penny Candle } \\
\text { Julius Caesar } \\
\text { A Town like Alice } \\
\text { Whisky Galore } \\
\text { Goodbye to All That } \\
\text { Breakfast at Tiffany's } \\
\text { The Decline and Fall of the Roman } \\
\text { Empire } \\
\text { The Name of Rose } \\
\text { Who's afraid of Virginia Woolf? } \\
\text { The Tin Drum } \\
\text { Toum }\end{array}$ & $\begin{array}{l}\text { Madame Bovary } \\
\text { Les Mille et une nuits } \\
\text { Crime et châtiment } \\
\text { Le Malade imaginaire } \\
\text { Zazie dans le métro } \\
\text { Le Petit prince } \\
\text { Don Quichotte } \\
\text { Le Désert des Tartares } \\
\text { Hamlet } \\
\text { Le Cousin Pons } \\
\text { Moby Dick } \\
\text { Les Canons de Navaron } \\
\text { Trois hommes dans un bateau } \\
\text { Le Vieil homme et la mer } \\
\text { L'Amant de Lady Chatterley } \\
\text { Le Mystère de la chambre jaune } \\
\text { Jules César } \\
\text { Voyage au bout de la nuit } \\
\text { Les Raisins de la colère } \\
\text { Roméo et Juliette } \\
\text { L'Homme invisible } \\
\text { L'Odyssée } \\
\text { La Toison d'or } \\
\text { L'Arrache-cour } \\
\text { Le Silence de la mer } \\
\text { Le Roi Lear } \\
\text { L'Assommoir } \\
\text { Le Rouge et le noir } \\
\text { Tristan et Iseut } \\
\text { Le Père Goriot } \\
\text { Le Fantôme de l'opéra }\end{array}$ \\
\hline
\end{tabular}

